\title{
机插粳稻氮磷钾吸收分配特征
}

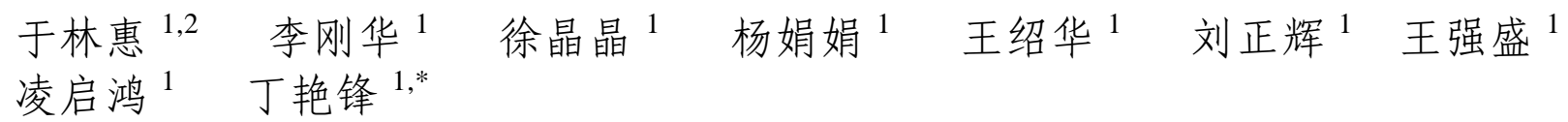

${ }^{1}$ 南京农业大学 / 农业部南方作物生理生态重点开放实验室, 江苏南京 210095; ${ }^{2}$ 江苏省农业机械管理局, 江苏南京 210024

摘 要: 调查研究 2008-2009 年江苏武进区漕桥(品种为武香粳 9 号)和前黄(品种为武运粳 7 号)等 2 个大于 $6.7 \mathrm{hm}^{2}$ 机插粳稻高产示范方, 探讨机插粳稻养分吸收分配特征, 并对高产精确定量施肥参数进行定量。结果表明, 高产机插 粳稻产量的 $80 \%$ 左右来自抽穗后的光合产物; 抽穗后叶片的干物质积累呈表观输出, 而茎鞘的干物质积累呈表观输 入; 抽穗后氮素的转运贡献率也主要来自叶片。机插粳稻对氮的吸收量随产量升高而增加, 增加量主要来自抽穗后; 随着产量提高, 抽穗至成熟期氮积累量和积累比例均上升, 对磷钾的吸收量上升但吸收比例却有下降的趋势; 成熟 期氮收获指数在 $0.51 \sim 0.61$ 之间, 磷收获指数在 0.75 左右, 钾收获指数接近 0.20 ; 高产机插粳稻百千克䊏粒需氮量为 $2.0 \sim 2.1 \mathrm{~kg}$, 氮磷钾吸收比例为 $2.0: 0.9: 1.4$ 。

关键词: 粳稻; 机插; 氮磷钾; 精确施肥; 参数

\section{Characteristics of Uptake of Nitrogen, Phosphorus, and Potassium and Parti- tioning in Mechanical Transplanting Japonica Rice}

YU Lin-Hui ${ }^{1,2}$, LI Gang-Hua ${ }^{1}$, XU Jing-Jing ${ }^{1}$, YANG Juan-Juan ${ }^{1}$, WANG Shao-Hua ${ }^{1}$, LIU Zheng-Hui ${ }^{1}$, WANG Qiang-Sheng ${ }^{1}$, LING Qi-Hong ${ }^{1}$, and DING Yan-Feng ${ }^{1, *}$

${ }^{1}$ Key Laboratory of Crop Physiology and Ecology in Southern China, Ministry of Agriculture / Nanjing Agricultural University, Nanjing 210095, China; ${ }^{2}$ Bureau of Agricultural Machinery Administration of Jiangsu Province, Nanjing 210024, China

\begin{abstract}
From 2008 to 2009, two more than 6.7 ha high-yield demonstration fields at Caoqiao (using cv. Wuxiangjing 9) and Qianhuang (using cv. Wuyunjing 7) in Wujin County, Jiangsu province, were used to investigate the nutrient uptake and partitionging characteristics of mechanical transplanting rice. The results showed that $80 \%$ of grain yield of mechanical transplanting rice came from the dry matter accumulation after heading, dry weight decreased in leaves while increased in stem sheath after heading. Leaves were also the main contributor of nitrogen translocation. Nitrogen uptake of mechanical transplanting rice increased with the yield, and the increased nitrogen uptake mainly originated from the nitrogen accumulation after heading. With the increasing yield, the accumulation and proportion of nitrogen from heading to maturity increased. At the same time, the uptake of $\mathrm{P}_{2} \mathrm{O}_{5}$ and $\mathrm{K}_{2} \mathrm{O}$ increased while the dry weight accumulation ratio reduced. The nitrogen harvest index was between 0.51 and 0.61 , the $\mathrm{P}_{2} \mathrm{O}_{5}$ harvest index was about 0.75 , and the $\mathrm{K}_{2} \mathrm{O}$ harvest index was close to 0.20 . For achieving high yield in mechanical transplanting rice, nitrogen requirement for $100 \mathrm{~kg}$ grains should be $2.0-2.1 \mathrm{~kg}$, and the optimum ratio of NPK should be $2.0: 0.9$ : 1.4 .
\end{abstract}

Keywords: Japonica; Mechanical transplanting rice; Nitrogen, Phosphorus, and Potassium; Parameters of precise and quantitative nutrient application; Index

随着农村劳动力的大量转移和老龄化现象的加 剧, 水稻生产迫切需要发展以机插积为主的机械种 植技术, 以适应稻农对现代稻作技术的要求, 稳定
水稻种植面积和提高水稻产量 ${ }^{[1-2]}$ 。2010 年江苏机插 秧面积 70 万公顷, 占水稻种植总面积的 $33 \%$, 机插 已成为主要的稻作栽培方式之一。提高机插稻产量,

本研究由国家星火计划项目(2010GA690010), 江苏农业自主创新项目(CX11, 2011), 引进国际先进农业科学技术计划(948 计划)重点 项目(2011-G18)资助。

* 通讯作者(Corresponding author): 丁艳锋, E-mail: dingyf@njau.edu.cn, Tel: 025-84395066

Received(收稿日期): 2011-05-10; Accepted(接受日期): 2011-12-19; Published online(网络出版日期): 2012-02-13.

URL: http://www.cnki.net/kcms/detail/11.1809.S.20120213.1103.001.html 
对稳定水稻单产具有决定性意义。研究不同施肥条 件下水稻植株对氮、磷、钾的吸收、积累和分配特 征, 有助于了解施肥对水稻产量形成的影响, 为水 稻的合理施肥提供依据。水稻对氮、磷、钾的吸收 和利用受品种特性 ${ }^{[3-4]}$ 、水肥管理 ${ }^{[5-9]}$ 、栽培措施 ${ }^{[10-11]}$ 及环境条件 ${ }^{[12]}$ 等因素的综合影响。以往的研究主要 针对常规手栽方式, 而针对机插稻作方式的研究相 对缺乏。本研究以江苏典型机插粳稻高产大于 $6.7 \mathrm{hm}^{2}$ 示范方为对象进行调查研究, 探讨机插粳稻养分吸 收分配特征, 并确定高产精确定量施肥参数, 以期 为机插粳稻高产栽培提供依据。

\section{1 材料与方法}

\section{1 试验设计}

2008-2009 年选择江苏武进区漕桥和前黄各 1 个大于 $6.7 \mathrm{hm}^{2}$ 高产示范方。土质均为黄壤土, 漕桥 土壤含全氮 $2.1 \mathrm{~g} \mathrm{~kg}^{-1}$, 有机质 $3.51 \%$, 速效钾 87.6 $\mathrm{mg} \mathrm{kg}^{-1}$, 有效磷 $8.82 \mathrm{mg} \mathrm{kg}^{-1}$; 前黄土壤含全氮 2.4 $\mathrm{g} \mathrm{kg}^{-1}$, 有机质 $3.58 \%$, 速效钾 $99.5 \mathrm{mg} \mathrm{kg}^{-1}$, 有效磷 $22.6 \mathrm{mg} \mathrm{kg}^{-1}$ 。种植品种分别为武香粳 9 号和武运粳 7 号, 均属早熟晚粳稻, 株高 $95 \mathrm{~cm}$ 左右, 生育期 $150 \mathrm{~d}$ 左右, 主茎总叶龄 17 18 叶, 伸长节间数 6 个。 示范方灌排条件良好, 常年水稻产量为 $8 \sim 9 \mathrm{t} \mathrm{hm}^{-2}$ 。 基本苗、株距、肥料运筹、水分管理等按照高产设 计方案实施。

方案根据品种总叶龄数 $(\mathrm{N})$ 、伸长节间数 $(\mathrm{n}) 、$ 移 栽积苗叶龄 $(\mathrm{SN})$ 以及目标穗数确定基本苗为 85 株 $\mathrm{m}^{-2}$, 行距 $30 \mathrm{~cm}$, 株距 $11.7 \mathrm{~cm}$, 每穴 3 苗左右。氮 肥用量 $180 \mathrm{~kg} \mathrm{hm}^{-2}$, 氮肥运筹按基藍肥: 穗肥 $=6$ : 4, 穗肥根据苗情适当调整, 群体偏大、叶色偏深的 田块施用量减少 $15 \mathrm{~kg} \mathrm{hm}^{-2}$, 反之增加 $15 \mathrm{~kg} \mathrm{hm}^{-2}$ 。 磷肥 $\left(\mathrm{P}_{2} \mathrm{O}_{5}, 90 \mathrm{~kg} \mathrm{hm}^{-2}\right)$ 作基肥施用，钾肥 $\left(\mathrm{K}_{2} \mathrm{O}, 150\right.$ $\mathrm{kg} \mathrm{hm}^{-2}$ )作基肥和拔节肥各半。移栽活棵期田间保 持浅水层, 当叶龄数达到 10.5 叶时开始排水晒田, 以后干湿交替灌溉; 移栽后 $7 \mathrm{~d}$ 施除草剂, 及时防 治病虫害。每个示范方选 5 块田设置空白区, 面积 均为 $60 \mathrm{~m}^{2}$, 空白区除不使用氮肥外, 其他同高产 方案。

\section{2 测定项目及方法}

每示范方各选取 15 块田, 每块田分别于移栽 期、有效分菜临界叶龄期 (N-n 叶龄期)、拔节期 $(n-2$ 叶龄期)、抽穗期、成熟期按 5 点取样法取 5 点, 每 点调查茎藍数 100 穴, 根据全田平均数取代表性样 5
穴, 每点 1 穴。样品按叶片、茎鞘、穗分离, 测定干 重、氮磷钾含量。用 $\mathrm{H}_{2} \mathrm{SO}_{4}-\mathrm{H}_{2} \mathrm{O}_{2}$ 消煮, 全自动凯氏 定氮仪测全氮; 钒钼黄比色法测全磷; 火焰光度法 测全钾 ${ }^{[13]}$ 。按 $14.5 \%$ 水分折算实产。2 年结果趋势 一致, 因此本文主要以 2008 年结果进行分析。

\section{3 参数计算与统计分析}

花后物质积累量 $\left(\mathrm{t} \mathrm{hm}{ }^{-2}\right)=$ 成熟期总干重-抽穗 期总干重

花后物质积累比例(\%)=花后积累量/籽粒干重 $\times 100$

氮(磷、钾)阶段积累量 $\left(\mathrm{kg} \mathrm{hm}^{-2}\right)=$ 某生育阶段单 位面积植株氮(磷、钾)的积累量

氮(磷、钾)收获指数=成熟期籽粒氮(磷、钾)积 累量/植株氮(磷、钾)总积累量

氮(磷、钾)转运量 $\left(\mathrm{t} \mathrm{hm}^{-2}\right)=$ 抽穗期叶、茎鞘氮 (磷、钾)积累量一成熟期叶、茎鞘氮(磷、钾)积累量

转运率 $(\%)=$ 氮( 磷、钾)转运量/抽穗期叶、茎鞘 氮(磷、钾)积累量 $\times 100 \%$

转运贡献率 $(\%)=$ 氮( 磷、钾)转运量/抽穗至成熟 期穗部氮(磷、钾)素积累总量 $\times 100 \%$

百千克籽粒吸氮(磷、钾)量 $\left(\mathrm{kg} 100 \mathrm{~kg}^{-1}\right)=$ 成熟期 氮(磷、钾)积累量/籽粒产量 $\times 100$

示范方产量分成 7.5 9.0 t hm${ }^{-2}, 9.0 \sim 10.5 \mathrm{t} \mathrm{hm}^{-2}$, 10.5 12.0 t hm${ }^{-2}$ 三个等级分级比较。用 Microsoft Excel 整理数据, 用 SPSS16.0 for Windows 进行方差 分析和作图。

\section{2 结果与分析}

2.1 机插粳稻示范方不同产量等级干物质积累 动态比较

表 1 表明, 示范方调查田块产量分布在 7.5 $12.0 \mathrm{t} \mathrm{hm}^{-2}$ 之间。为便于比较, 将 2 个示范方按产量 高低分成 7.5 9.0、9.0 10.5 以及 $10.5 \sim 12.0 \mathrm{t} \mathrm{hm}^{-2} 3$ 个 等级。其中漕桥示范方 3 个产量等级的田块数分别 为 $4 、 8$ 和 3 块, 而前黄示范方的田块数分别为 $2 、 9$ 和 4 块。

表 2 表明, 不同示范方各生育期干物质积累、 成熟期干物质量以及花后比例差异不显著。抽穗前 各时期不同产量等级间干物质积累量差异不显著, 成熟期干物质量差异极显著。示范方和产量等级互 作效应对不同阶段干物质积累量影响不显著。

不同示范方各生育期干物质积累比例差异不显 著。而不同产量等级仅在 $\mathrm{N}-\mathrm{n}$ 至拔节期差异不显著, 
表 1 机插粳稻示范方不同等级的产量及其构成因子比较

Table 1 Comparison of yield and its components in different treatments of machine-transplanted japonica rice

\begin{tabular}{|c|c|c|c|c|c|c|c|c|}
\hline $\begin{array}{c}\text { 示范方 } \\
\text { Site }\end{array}$ & $\begin{array}{c}\text { 等级 } \\
\text { Yield level } \\
\left.(\mathrm{t} \mathrm{hm})^{-2}\right)\end{array}$ & $\begin{array}{c}\text { 穗数 } \\
\text { Panicles per } \\
\mathrm{m}^{2}\end{array}$ & $\begin{array}{l}\text { 每穗粒数 } \\
\text { Spikelets per } \\
\text { panicle }\end{array}$ & $\begin{array}{c}\text { 总渘花 } \\
\text { Spikelets per } \\
\mathrm{m}^{2}\left(\times 10^{3}\right)\end{array}$ & $\begin{array}{c}\text { 结实率 } \\
\text { Seed setting } \\
\text { rate (\%) }\end{array}$ & $\begin{array}{c}\text { 粒重 } \\
\text { Grain weight } \\
\text { (mg) }\end{array}$ & $\begin{array}{c}\text { 平均产量 } \\
\text { Yield } \\
\left(\mathrm{t} \mathrm{hm}^{-2}\right)\end{array}$ & $\begin{array}{c}\text { 田块数 } \\
\text { No. of } \\
\text { plot }\end{array}$ \\
\hline \multirow{3}{*}{$\begin{array}{c}\text { 漕桥 } \\
\text { Caoqiao }\end{array}$} & $7.5-9.0$ & 387.6 & 106.3 & 41.2 & 91.7 & 23.3 & 8.8 & 4 \\
\hline & 9.0-10.5 & 388.7 & 112.3 & 43.5 & 91.1 & 24.1 & 9.5 & 8 \\
\hline & 10.5-12.0 & 375.1 & 123.8 & 46.4 & 92.8 & 24.8 & 10.7 & 3 \\
\hline \multirow{3}{*}{$\begin{array}{c}\text { 前黄 } \\
\text { Qianhuang }\end{array}$} & $7.5-9.0$ & 281.3 & 127.7 & 35.9 & 90.4 & 26.6 & 8.6 & 2 \\
\hline & $9.0-10.5$ & 323.7 & 128.5 & 41.5 & 90.4 & 25.5 & 9.6 & 9 \\
\hline & $10.5-12.0$ & 328.2 & 135.2 & 44.3 & 91.0 & 26.7 & 10.7 & 4 \\
\hline
\end{tabular}

表 2 机插粳稻示范方不同产量等级干物质积累比较

Table 2 Comparison of rice dry matter accumulation in different treatments of machine-transplanted japonica rice

\begin{tabular}{|c|c|c|c|c|c|c|c|c|c|}
\hline \multirow{2}{*}{$\begin{array}{l}\text { 等级 } \\
\text { Rating } \\
\left(\mathrm{t} \mathrm{hm}^{-2}\right)\end{array}$} & \multicolumn{2}{|c|}{$\begin{array}{l}\text { 移栽至 } \mathrm{N}-\mathrm{n} \text { 叶龄期 } \\
\text { Transplanting to N-n }\end{array}$} & \multicolumn{2}{|c|}{$\begin{array}{c}\text { N-n 叶龄期至拔节期 } \\
\text { N-n to Jointing } \\
\end{array}$} & \multicolumn{2}{|c|}{$\begin{array}{c}\text { 拔节期至抽穗期 } \\
\text { Jointing to Heading }\end{array}$} & \multicolumn{2}{|c|}{$\begin{array}{c}\text { 抽穗期至成熟期 } \\
\text { Heading to Maturity }\end{array}$} & \multirow{2}{*}{$\begin{array}{c}\text { 花后比例 } \\
\text { Accumulation } \\
\text { ratio after } \\
\text { flowering (\%) }\end{array}$} \\
\hline & $\begin{array}{c}\text { 积累量 } \\
\text { Biomass } \\
\left(\mathrm{t} \mathrm{hm}^{-2}\right)\end{array}$ & $\begin{array}{c}\text { 比例 } \\
\text { Ratio } \\
(\%)\end{array}$ & $\begin{array}{c}\text { 积累量 } \\
\text { Biomass } \\
\left(\mathrm{t} \mathrm{hm}^{-2}\right)\end{array}$ & $\begin{array}{c}\text { 比例 } \\
\text { Ratio } \\
(\%)\end{array}$ & $\begin{array}{c}\text { 积累量 } \\
\text { Biomass } \\
\left(\mathrm{t} \mathrm{hm}^{-2}\right)\end{array}$ & $\begin{array}{l}\text { 比例 } \\
\text { Ratio } \\
(\%)\end{array}$ & $\begin{array}{c}\text { 积累量 } \\
\text { Biomass } \\
\left(\mathrm{t} \mathrm{hm}^{-2}\right)\end{array}$ & $\begin{array}{l}\text { 比例 } \\
\text { Ratio } \\
(\%)\end{array}$ & \\
\hline \multicolumn{10}{|l|}{ 漕桥 Caoqiao } \\
\hline $7.5-9.0$ & 1.4 & 8.8 & 2.8 & 18.2 & 6.7 & 44.5 & 4.4 & 28.5 & 57.0 \\
\hline $9.0-10.5$ & 1.6 & 9.5 & 2.9 & 17.1 & 7.1 & 41.4 & 5.6 & 32.0 & 67.1 \\
\hline $10.5-12.0$ & 1.5 & 8.1 & 2.1 & 11.3 & 7.6 & 40.9 & 7.4 & 39.8 & 78.8 \\
\hline 平均 Average & 1.5 & 8.8 & 2.6 & 15.3 & 7.1 & 42.6 & 5.8 & 33.4 & 67.6 \\
\hline \multicolumn{10}{|l|}{ 前黄 Qianhuang } \\
\hline $7.5-9.0$ & 1.6 & 10.3 & 3.1 & 19.5 & 5.7 & 36.2 & 5.3 & 34.0 & 69.6 \\
\hline $9.0-10.5$ & 1.5 & 7.7 & 3.2 & 16.4 & 6.3 & 32.2 & 6.4 & 43.8 & 76.0 \\
\hline $10.5-12.0$ & 1.6 & 6.6 & 3.3 & 15.3 & 6.2 & 26.2 & 7.5 & 51.9 & 79.9 \\
\hline 平均 Average & 1.6 & 8.2 & 3.2 & 17.0 & 6.1 & 31.5 & 6.4 & 43.3 & 75.2 \\
\hline \multicolumn{10}{|l|}{ 方差分析 Analysis of variance } \\
\hline 示范方 Treatments & ns & ns & ns & ns & ns & ns & ns & ns & ns \\
\hline 等级 Rating & ns & $* *$ & ns & ns & ns & $*$ & $* *$ & $* *$ & $* *$ \\
\hline 示范方×等级 Treat. ×rating & ns & $*$ & ns & ns & ns & ns & ns & ns & ns \\
\hline
\end{tabular}

$\mathrm{N}-\mathrm{n}$ : 有效分葟临界叶龄期; 花后比例(\%)=花后积累量/籽粒干重 $\times 100 \%$ 。表示在 0.05 水平差异显著; **表示在 0.01 水平差异显 著; ns: 表示差异不显著。

$\mathrm{N}-\mathrm{n}$ : Critical leaf-age for productive tillers. Accumulation ratio after flowering (\%)=dry matter accumulation after flowering/grain dry weight $\times 100 \%$. * Significant at the 0.05 probability level; ** Significant at the 0.01 probability level; ns: not significant.

其他各生育阶段差异均显著或极显著。随着产 量提高, 移栽至 $\mathrm{N}-\mathrm{n}$ 期和拔节至抽穗期 2 个生育阶 段干物质积累所占比例有下降的趋势, 而抽穗至成 熟期干物质积累所占比例极显著增加。示范方和产 量等级互作效应对移栽至 $\mathrm{N}-\mathrm{n}$ 期干物质积累比例有 显著影响, 对其他阶段干物质积累比例影响不显 著。两个示范方花后干物质积累率均随产量提高而 提高。当产量为 $10.5 ~ 12.0 \mathrm{t} \mathrm{hm}^{-2}$ 时, 漕桥示范方的 花后比为 $78.8 \%$, 而前黄示范方的花后比为 $79.9 \%$, 2 个示范方非常接近, 花后比例均在 $80 \%$ 左右。示范 方和产量等级互作效应对花后比例影响不显著。
随着生育进程, 不同产量等级后期干重差异加 大(图 1)。叶片的干物质在抽穗期后表现表观输出, 且随着产量的提高, 叶片的表观转运量有降低的趋 势, 2 个示范方表现一致。茎鞘干重在抽穗至成熟期 不仅没有降低, 反而有一定上升, 而且随着产量等 级的升高, 茎鞘干重增加量有上升的趋势, 漕桥示 范方茎鞘干重增加量从 $0.3 \mathrm{t} \mathrm{hm}^{-2}$ 提高到 $1.4 \mathrm{t} \mathrm{hm}^{-2}$, 前黄示范方茎鞘干重增加量从 $0.4 \mathrm{t} \mathrm{hm}^{-2}$ 提高到 $3.6 \mathrm{t}$ $\mathrm{hm}^{-2}$ 。表明机插粳稻抽穗后茎鞘的干物质积累表现 表观输入, 且随着产量升高, 表观输入量有增加的 趋势。 

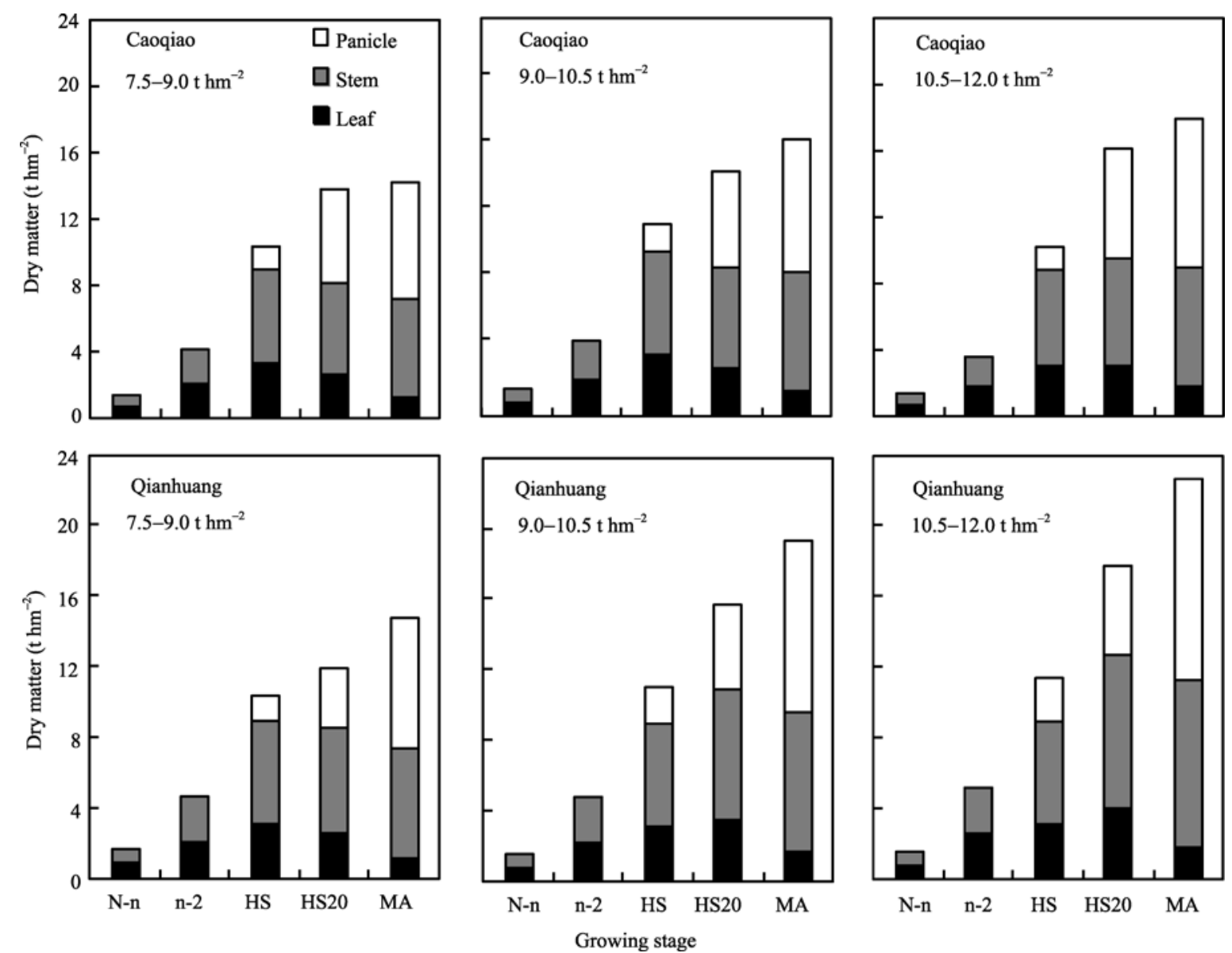

图 1 机插粳稻不同器官干物质积累动态

Fig. 1 Dry matter accumulation of different organs of machine-transplanted japonica rice N-n: 有效分葟临界叶龄期; n-2：拔节期; HS：抽穗期; HS20：抽穗后 20d; MA：成熟期。

$\mathrm{N}-\mathrm{n}$ : tiller critical leaf age; $\mathrm{n}-2$ : jointing stage; HS: heading stage; HS20: 20 days after heading stage; MA: maturity.

2.2 机插粳稻示范方不同产量等级氮磷钾积累 动态比较

表 3 表明, 不同产量等级间从移栽至抽穗期氮 积累量差异不显著, 但抽穗期氮积累量差异极显 著。随着产量提高, 抽穗至成熟期氮积累量显著增 加。最终成熟期总氮积累量差异极显著。示范方和 产量等级间对各生育阶段氮积累量互作效应不显 著。不同生育阶段氮积累比例与氮积累量有类似的 趋势。表明机插粳稻产量提高, 氮素积累量上升, 尤 其后期的优势明显。

为便于比较, 方便实际应用, 综合 2 个示范方 数据, 将产量分成 $<9 \mathrm{t} \mathrm{hm}^{-2}$ 和 $\geq 9 \mathrm{thm}^{-2}$ 两个产量等 级, 生育时期合并为移栽至 $\mathrm{N}-\mathrm{n} 、 \mathrm{~N}-\mathrm{n}$ 至抽穗期以 及抽穗至成熟期等 3 个阶段(图 2)。结果表明, 移栽 至 N-n 期, 钾素吸收比例最高, 达 30\%左右; 氮素 次之, 占 $20 \%$ 左右; 磷素最少, 仅占 $10 \%$ 左右; N-n
至抽穗期, 氮素和钾素吸收比例较大, 达 $60 \%$ 左右; 磷素略低, 占 $45 \%$ 左右。在抽穗至成熟期, 磷素吸 收比例最大, 达 $45 \%$ 左右; 氮素次之, 占 $20 \%$ 左右; 钾素最少, 占 $10 \%$ 左右。随着产量提高, 抽穗后氮 素积累的比例上升, 而磷钾吸收比例均有下降的趋 势。

2.3 机插粳稻示范方不同产量等级氮磷钾分配 比较

表 4 表明, 水稻抽穗至成熟期叶片氮转运率最 高, 漕桥示范方平均转运量为 $47.2 \mathrm{~kg} \mathrm{hm}^{-2}$, 转运率 达 $70.8 \%$, 前黄示范方平均转运量为 $34.7 \mathrm{~kg} \mathrm{hm}^{-2}$, 转运率为 $61.3 \%$ 。示范方和产量等级间叶片转运量 和转运率均未达到显著水平, 但示范方和产量等级 互作对叶片的转运率影响显著。而茎鞘氮素表观转 运量总体呈负值, 表现对氮素的再积累。漕桥示范 方平均再积累量为 $6.4 \mathrm{~kg} \mathrm{hm}^{-2}$, 输入率为 $13.3 \%$, 前 
黄示范方平均再积累量为 $13.2 \mathrm{~kg} \mathrm{hm}^{-2}$, 反转运率达 $20.6 \%$ 。产量等级间以及示范方与产量等级的互作对 穗部氮素增加量影响不显著, 而互作关系对氮转运 贡献率影响显著。

养分的收获指数是指水稻吸收的养分被转运 到水稻籽粒中的百分数, 即养分在籽粒中的分配 比例。表 5 表明, 漕桥示范方氮、磷、钾的收获指 数分别为 $0.539 \sim 0.594 、 0.731 \sim 0.777$ 和 $0.164 \sim 0.191$,
随着产量水平的升高, 氮收获指数呈上升趋势, 而磷、钾收获指数呈下降趋势。前黄示范方的氮、 磷、钾的收获指数分别为 $0.510 \sim 0.615 、 0.733 \sim 0.748$ 和 $0.181 \sim 0.209$ 。随着产量水平的升高, 氮收获指 数呈下降趋势, 而磷、钾收获指数规律性不明显。 方差分析表明，示范方、产量等级间以及示范方与 等级的互作，对氮、磷、钾的收获指数影响均不显 著。

表 3 机插粳稻示范方不同产量等级氮素积累比较

Table 3 Comparison of $\mathrm{N}$ accumulation in different sites of machine- transplanted japonica rice

\begin{tabular}{|c|c|c|c|c|c|c|c|c|c|}
\hline \multirow{2}{*}{ 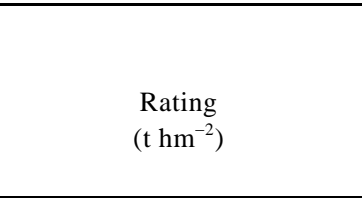 } & \multicolumn{2}{|c|}{$\begin{array}{l}\text { 移栽至 N-n 叶龄期 } \\
\text { Transplanting to N-n }\end{array}$} & \multicolumn{2}{|c|}{$\begin{array}{c}\mathrm{N}-\mathrm{n} \text { 叶龄期至拔节期 } \\
\mathrm{N}-\mathrm{n} \text { to jointing }\end{array}$} & \multicolumn{2}{|c|}{$\begin{array}{c}\text { 拔节期至抽穗期 } \\
\text { Jointing to heading }\end{array}$} & \multicolumn{2}{|c|}{$\begin{array}{c}\text { 抽穗期至成熟期 } \\
\text { Heading to maturity }\end{array}$} & \multirow{2}{*}{$\begin{array}{c}\text { 成熟期 } \\
\text { Maturity } \\
\left(\mathrm{kg} \mathrm{hm}^{-2}\right)\end{array}$} \\
\hline & $\begin{array}{l}\text { 积累量 } \\
\text { Biomass } \\
\left(\mathrm{t} \mathrm{hm}^{-2}\right)\end{array}$ & $\begin{array}{c}\text { 比例 } \\
\text { Ratio } \\
(\%)\end{array}$ & $\begin{array}{c}\text { 积累量 } \\
\text { Biomass } \\
\left(\mathrm{t} \mathrm{hm}^{-2}\right)\end{array}$ & $\begin{array}{c}\text { 比例 } \\
\text { Ratio } \\
(\%)\end{array}$ & $\begin{array}{c}\text { 积累量 } \\
\text { Biomass } \\
\left(\mathrm{t} \mathrm{hm}^{-2}\right)\end{array}$ & $\begin{array}{c}\text { 比例 } \\
\text { Ratio } \\
(\%)\end{array}$ & $\begin{array}{c}\text { 积累量 } \\
\text { Biomass } \\
\left(\mathrm{t} \mathrm{hm}^{-2}\right)\end{array}$ & $\begin{array}{c}\text { 比例 } \\
\text { Ratio } \\
(\%)\end{array}$ & \\
\hline \multicolumn{10}{|l|}{ 漕桥 Caoqiao } \\
\hline $7.5-9.0$ & 38.0 & 22.9 & 43.9 & 29.1 & 53.4 & 33.3 & 29.6 & 14.6 & 164.8 \\
\hline $9.0-10.5$ & 36.4 & 18.9 & 54.7 & 28.5 & 70.2 & 36.6 & 31.2 & 15.9 & 192.4 \\
\hline $10.5-12.0$ & 51.3 & 23.1 & 55.6 & 25.0 & 71.6 & 32.2 & 44.0 & 19.8 & 222.5 \\
\hline 平均 Average & 41.9 & 21.6 & 51.4 & 27.5 & 65.0 & 34.1 & 34.9 & 16.8 & 193.2 \\
\hline \multicolumn{10}{|l|}{ 前黄 Qianhuang } \\
\hline $7.5-9.0$ & 33.7 & 17.5 & 49.7 & 25.3 & 66.7 & 34.2 & 46.1 & 23.0 & 196.2 \\
\hline $9.0-10.5$ & 30.2 & 13.9 & 55.3 & 25.6 & 64.8 & 29.8 & 66.9 & 30.8 & 217.1 \\
\hline $10.5-12.0$ & 28.8 & 12.7 & 58.9 & 30.6 & 50.2 & 22.1 & 77.8 & 34.6 & 225.7 \\
\hline 平均 Average & 30.9 & 14.7 & 54.6 & 27.2 & 60.5 & 28.7 & 63.6 & 29.4 & 213.0 \\
\hline \multicolumn{10}{|l|}{ 方差分析 Analysis of variance } \\
\hline 示范方 Treatment & $*$ & $*$ & ns & ns & ns & ns & $*$ & $*$ & $*$ \\
\hline 等级 Rating & ns & ns & ns & ns & ns & ns & $* *$ & $*$ & $* *$ \\
\hline 示范方 ×等级 Treat. $\times$ rating & ns & ns & ns & ns & ns & ns & ns & ns & ns \\
\hline
\end{tabular}

* 表示在 0.05 水平差异显著; **表示在 0.01 水平差异显著; ns: 表示差异不显著。

* Significant at the 0.05 probability level; ** Significant at the 0.01 probability level; ns: not significant.
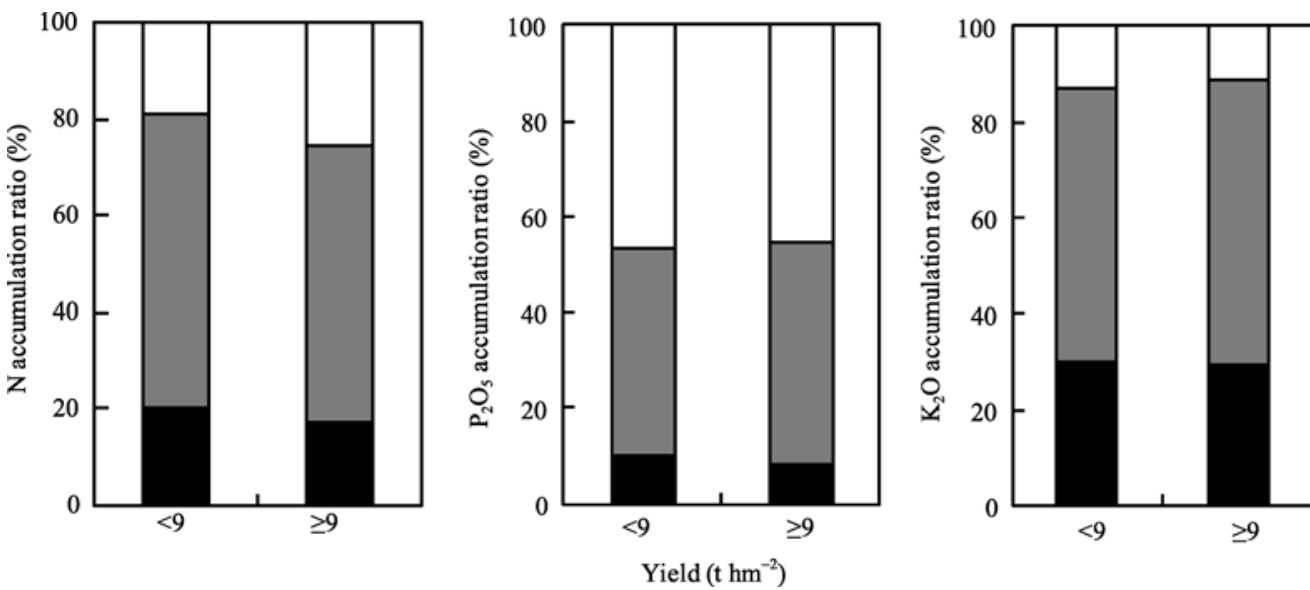

$\square$ MA-HS

HS-(N-n)

(N-n)-Ts

图 2 机插粳稻不同生育阶段氮磷钾积累比较

Fig. 2 Comparison of NPK accumulation at different stages of machine-transplanted japonica rice TS: 移栽期; N-n: 有效分薛临界叶龄期; HS: 抽穗期; MA：成熟期。

TS: transplanting stage; $\mathrm{N}-\mathrm{n}$ : tiller critical leaf age; HS: heading stage; MA: maturity. 
表 4 机插粳稻示范方不同产量等级抽穗至成熟期叶片及茎鞘氮的转运

Table $4 \mathrm{~N}$ translocation in leaves and stem-sheathes from heading to maturity under different yield levels in different sites

\begin{tabular}{|c|c|c|c|c|c|c|}
\hline \multirow{2}{*}{$\begin{array}{l}\text { 等级 } \\
\text { Rating } \\
\left(\mathrm{t} \mathrm{hm}^{-2}\right)\end{array}$} & \multicolumn{2}{|c|}{ 叶片 Leaf } & \multicolumn{2}{|c|}{ 茎鞘 Stem-sheath } & \multirow{2}{*}{$\begin{array}{c}\text { 穗部氮增加量 } \\
\mathrm{N} \text { increase in } \\
\text { panicle } \\
\left(\mathrm{kg} \mathrm{hm}^{-2}\right)\end{array}$} & \multirow[b]{2}{*}{$\begin{array}{c}\text { 氮转运贡献率 } \\
\text { NTCRV } \\
(\%)\end{array}$} \\
\hline & $\begin{array}{c}\text { 氮转运量 } \\
\mathrm{NT} \\
\left(\mathrm{kg} \mathrm{hm}^{-2}\right)\end{array}$ & $\begin{array}{c}\text { 氮转运率 } \\
\text { NTE } \\
(\%)\end{array}$ & $\begin{array}{c}\text { 氮转运量 } \\
\mathrm{NT} \\
\left(\mathrm{kg} \mathrm{hm}^{-2}\right)\end{array}$ & $\begin{array}{c}\text { 氮转运率 } \\
\text { NTE } \\
(\%)\end{array}$ & & \\
\hline \multicolumn{7}{|l|}{ 漕桥 Caoqiao } \\
\hline $7.5-9.0$ & 40.3 & 65.3 & 17.0 & 20.0 & 72.0 & 94.3 \\
\hline $9.0-10.5$ & 51.0 & 74.3 & -13.9 & -24.4 & 72.5 & 49.2 \\
\hline $10.5-12.0$ & 37.0 & 58.0 & -25.0 & -36.0 & 72.0 & 16.0 \\
\hline 平均 Average & 47.2 & 70.8 & -6.4 & -13.3 & 72.3 & 59.0 \\
\hline \multicolumn{7}{|l|}{ 前黄 Qianhuang } \\
\hline $7.5-9.0$ & 36.5 & 66.5 & -19.0 & -31.5 & 86.5 & 19.5 \\
\hline $9.0-10.5$ & 32.8 & 58.3 & -13.7 & -21.6 & 80.1 & 19.8 \\
\hline $10.5-12.0$ & 38.0 & 65.3 & -9.3 & -13.0 & 93.5 & 30.8 \\
\hline 平均 Average & 34.7 & 61.3 & -13.2 & -20.6 & 84.5 & 22.7 \\
\hline \multicolumn{7}{|l|}{ 方差分析 Analysis of variance } \\
\hline 示范方 Treatment & ns & ns & ns & ns & $*$ & $*$ \\
\hline 等级 Rating & ns & ns & ns & ns & ns & ns \\
\hline 示范方×等级 Treat. $\times$ rating & ns & * & ns & ns & ns & * \\
\hline
\end{tabular}

NT: 氮转运量; NTE: 氮转运率; NTCRV: 氮转运贡献率。* 表示在 0.05 水平差异显著; ns: 表示差异不显著。

NT: N translocation; NTE: N transportation efficiency; NTCRV: N translocation conversion rate of vegetative organ. ${ }^{*}$ Significant at the 0.05 probability level; ns: not significant.

表 5 机插粳稻氮素、磷素、钾素的收获指数

Table $5 \mathrm{~N}$, P, and $\mathrm{K}$ harvest index of machine-transplanted rice

\begin{tabular}{|c|c|c|c|}
\hline $\begin{array}{c}\text { 等级 } \\
\text { Rating }\left(\mathrm{t} \mathrm{hm}{ }^{-2}\right) \\
\end{array}$ & $\begin{array}{c}\text { 氮收获指数 } \\
\mathrm{N} \text { harvest index }\end{array}$ & $\begin{array}{c}\text { 磷收获指数 } \\
\text { P harvest index }\end{array}$ & $\begin{array}{c}\text { 钾收获指数 } \\
\text { K harvest index }\end{array}$ \\
\hline \multicolumn{4}{|l|}{ 漕桥 Caoqiao } \\
\hline 7.5-9.0 & 0.539 & 0.777 & 0.191 \\
\hline $9.0-10.5$ & 0.558 & 0.753 & 0.174 \\
\hline $10.5-12.0$ & 0.594 & 0.731 & 0.164 \\
\hline 平均 Average & 0.555 & 0.758 & 0.178 \\
\hline \multicolumn{4}{|l|}{ 前黄 Qianhuang } \\
\hline $7.5-9.0$ & 0.615 & 0.733 & 0.181 \\
\hline $9.0-10.5$ & 0.573 & 0.748 & 0.209 \\
\hline $10.5-12.0$ & 0.510 & 0.744 & 0.184 \\
\hline 平均 Average & 0.562 & 0.745 & 0.199 \\
\hline \multicolumn{4}{|l|}{ 方差分析 Analysis of variance } \\
\hline 示范方 Treatment & ns & ns & ns \\
\hline 等级 Rating & ns & ns & ns \\
\hline 示范方×等级 Treat.×rating & ns & ns & ns \\
\hline
\end{tabular}

ns 表示差异不显著。ns: not significant.

\section{4 机插粳稻百千克籽粒养分需求量}

百千克籽粒养分需求量常作为水稻养分需求和 高产栽培定量指标 ${ }^{[14]}$ 。表 6 表明, 机插粳稻百千克 籽粒氮磷钾需求量与产量均呈开口向下的二次曲线 关系, 方程拟合达极显著水平。回归分析表明, 漕桥 示范方机插粳稻百千克籽粒需氮量为 $2.02 \mathrm{~kg} 100$ $\mathrm{kg}^{-1}$, 需钾量为 $0.90 \mathrm{~kg} 100 \mathrm{~kg}^{-1}$, 需磷量为 $1.38 \mathrm{~kg}$ $100 \mathrm{~kg}^{-1}$ 。前黄示范方机插粳稻百千克籽粒需氮量为

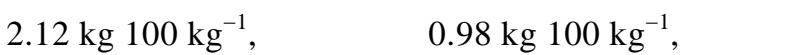

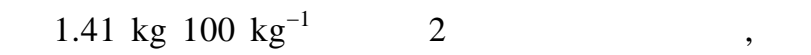
苏高产机插粳稻百千克籽粒需氮量为 $2.0 \sim 2.1 \mathrm{~kg}$ $\mathrm{hm}^{-2}$, 氮磷钾需求比例为 $2.0 ： 0.9 ： 1.4$ 。 
表 6 机插粳稻百千克籽粒养分需要量

Table 6 Nutrition uptake of $100 \mathrm{~kg}$ grain in machine-transplanted rice

\begin{tabular}{|c|c|c|c|}
\hline $\begin{array}{c}\text { 养分 } \\
\text { Nutrient }\end{array}$ & $\begin{array}{l}\text { 与产量的回归方程 } \\
\text { Regression equation }\end{array}$ & $\begin{array}{c}\text { 最适值 } \\
\text { Suitable value }\left(\mathrm{kg} 100 \mathrm{~kg}^{-1}\right)\end{array}$ & $R^{2}$ \\
\hline \multicolumn{4}{|l|}{ 漕桥 Caoqiao } \\
\hline $\mathrm{N}$ & $y=-3.8092 x^{2}+15.414 x-5.8279$ & 2.02 & $0.3904^{* *}$ \\
\hline $\mathrm{P}_{2} \mathrm{O}_{5}$ & $y=-8.1686 x^{2}+14.733 x+3.2681$ & 0.90 & $0.4068^{* *}$ \\
\hline $\mathrm{K}_{2} \mathrm{O}$ & $y=-6.54 x^{2}-18.02 x+9.6781$ & 1.38 & $0.4753^{* *}$ \\
\hline \multicolumn{4}{|c|}{ 前黄 Qianhuang } \\
\hline $\mathrm{N}$ & $y=-10.911 x^{2}+46.284 x-38.843$ & 2.12 & $0.5303^{* *}$ \\
\hline $\mathrm{P}_{2} \mathrm{O}_{5}$ & $y=-18.785 x^{2}+36.859 x-7.9735$ & 0.98 & $0.2806^{* *}$ \\
\hline $\mathrm{K}_{2} \mathrm{O}$ & $y=-0.4585 x^{2}+1.2906 x+8.7978$ & 1.41 & $0.4274^{* *}$ \\
\hline
\end{tabular}

**表示在 0.01 水平差异显著。 ${ }^{* *}$ Significant at the 0.01 probability level.

\section{5 机插粳稻土壤养分供应特点}

用不施氮空白区测得的稻谷养分吸收量, 反映 了土壤的养分综合供应能力 ${ }^{[14]}$ 。不施肥处理的空白

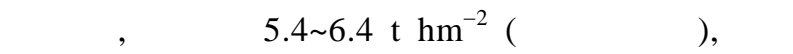
磷、钾的基础供应量(图 3), 平均分别为 113.0、46.8

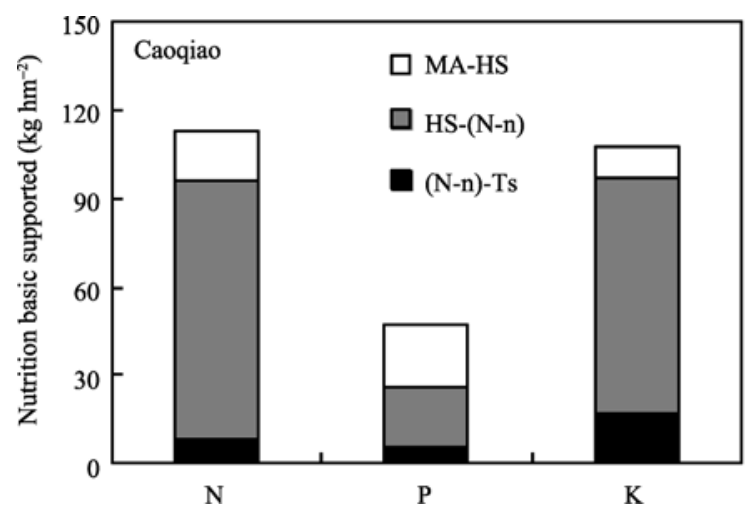

和 $107.0 \mathrm{~kg} \mathrm{hm}^{-2}$ 。前黄示范方不施肥处理的空白区 产量在 5.9 6.8 t hm${ }^{-2}$ 之间, 比漕桥点略高, 氮、磷、 钾的基础供应量也略高于漕桥, 平均分别为 128.0 、 54.4 和 $110.0 \mathrm{~kg} \mathrm{hm}^{-2}$ 。相对于高产田, 空白区氮、 磷、钾在 $\mathrm{N}-\mathrm{n}$ 至抽穗期吸收比例更高。

图 3 机插粳稻不同生育阶段基础养分供应量比较

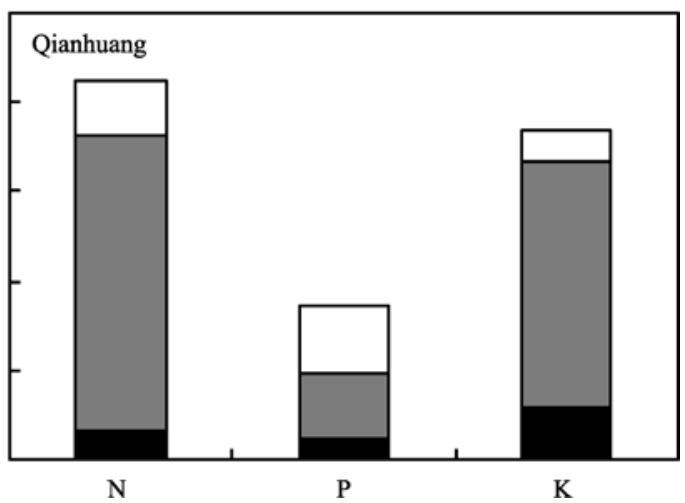

Fig. 3 Comparison of basic nutrition accumulation at different stages in machine-transplanted rice TS: 移栽期; N-n: 有效分藮临界叶龄期; HS: 抽穗期; MA：成熟期。

TS: transplanting stage; $\mathrm{N}-\mathrm{n}$ : tiller critical leaf age; HS: heading stage; MA: maturity.

\section{3 讨论}

\section{1 高产机插粳稻干物质积累与转运特征}

水稻籽粒产量取决于光合生产能力和光合产物 的运转和分配。关于干物质积累和分配, 不同研究 者得出的结论差异较大, 陈温福等 ${ }^{[16]}$ 和张洪松 ${ }^{[17]}{ }^{\text {等 }}$ 认为高产品种干物质生产优势在抽穗前。Ying ${ }^{[18]}$ 等 和杨惠杰等 ${ }^{[19-21]}$ 研究认为高产水稻的干物质积累优 势在中、后期。凌启鸿等 ${ }^{[22-23]}$ 和张洪程等 ${ }^{[24]}$ 认为高 产水稻的特点是后期的积累量高, 邹应斌等 ${ }^{[25]}$ 则认 为水稻每个生长时期都应有合适的比例。本研究结 果表明, 机插高产水稻的干物质积累优势来自抽穗 后。高产机插粳稻产量的 $80 \%$ 左右来自抽穗后的光合
产物, 这与超级粳稻高产方的研究结果一致 ${ }^{[15-18]}$ 。

另外, 本研究发现成熟期茎鞘的干重比抽穗期 高, 表明茎鞘干物质呈表观输入态势, 这是否有利 于提高机插䊅稻的抗倒能力 ${ }^{[26]}$, 有待进一步研究。

\section{2 高产机插粳稻氮磷钾吸收与分配特征}

氮磷钾的吸收和分配对物质生产和产量形成至 关重要。本研究结果表明, 机插粳稻百千克籽粒氮 磷钾需求量与产量均呈开口向下的二次曲线关系, 通过方程拟合, 高产机插粳稻百千克籽粒需氮量为 $2.0 \sim 2.1 \mathrm{~kg}^{2} 100 \mathrm{~kg}^{-1}$, 氮磷钾比例为 $2.0: 0.9: 1.4$ 。 与其他高产栽培方式相比 ${ }^{[9]}$, 磷素的吸收比例增加, 而钾素的吸收比例下降较明显。

随着产量升高，机插粳稻 $\mathrm{N}-\mathrm{n}$ 抽穗期氮素吸 
收比例降低、抽穗后吸收比例显著升高。这符合高 产栽培中粳稻对养分吸收具有生育前期较低、中后 期较高的特点 ${ }^{[9]}$ 。与氮素吸收不同的是, 产量提高后, 磷钾的吸收量虽有所增加, 吸收比例却有下降的趋 势。

研究结果表明, 机插粳稻叶片的氮素转运率较 高, 而茎鞘在抽穗后呈表观输入。机插粳稻氮收获 指数为 $0.510 ~ 0.610$, 磷收获指数为 0.75 左右, 钾收 获指数接近 0.20 。与其他栽培方式的表现不同 ${ }^{[1,6,27]}$, 机插粳稻穗部磷钾的分配比例相当, 而氮素的分配 比例较低, 且不同产量等级之间氮磷钾的收获指数 差异不显著。

\section{3 高产机插粳稻氮素定量参数研究}

按照水稻精确定量栽培基本原理 ${ }^{[28]}$, 水稻氮磷 钾的施用中首要的是确定氮的施用总量, 然后按适 宜的氮磷钾比例确定磷钾的施用量。水稻精确定量 施氮的方法是以产量等级和品种类型定量确定参数 值 ${ }^{[14]}$ 。此定量方法已开始大面积应用, 并取得了高 产高效的双重效果 ${ }^{[29-30]}$ 。

精确定量施氮主要是确定基础供氮量、目标产 量需氮量和氮素利用率。其中基础供氮量反映了品 种、土壤和生态条件的综合 ${ }^{[14]}$ 。本研究平均基础供 氮量漕桥示范方为 $113.0 \mathrm{~kg} \mathrm{hm}^{-2}$, 前黄示范方为 $128.0 \mathrm{~kg} \mathrm{hm}^{-2}$ 。高产田的单位籽粒需氮量可以通过 高产实践经验, 或与高产田的产量回归获得。本研 究通过方程拟合, 高产机插粳稻百千克籽粒需氮量为 2.0 2.1 $\mathrm{kg} \mathrm{hm}^{-2}$, 与常规高产粳稻结果基本一致 ${ }^{[25]}$ 。由 于氮素利用率受氮肥管理方式的影响较大 ${ }^{[28-34]}$, 关 于机插稻氮素利用率的问题, 将另文讨论。

\section{4 结论}

机插粳稻产量提高源自抽穗后氮素积累量和比 例上升, 磷钾吸收量增加, 但比例却有下降的趋 势。机插粳稻氮素的转运贡献率主要来自叶片, 而 茎鞘抽穗后呈表观输入。成熟期氮收获指数在 0.51 0.61 之间, 磷收获指数在 0.75 左右, 钾收获指 数接近 0.20 。高产机插粳稻百千克籽粒需氮量为 $2.0 \sim 2.1 \mathrm{~kg} \mathrm{hm}^{-2}$, 氮磷钾吸收比例为 $2.0 ： 0.9 ： 1.4$ 。

\section{References}

[1] Zhu D-F(朱德峰), Chen H-Z(陈惠哲). Development of mechanical-transplanting rice and food safety. China Rice (中国 稻米), 2009, (6): 4-7 (in Chinese)
[2] Zhu D-F(朱德峰), Cheng S-H(程式华), Zhang Y-P(张玉屏), Lin $\mathrm{X}-\mathrm{Q}$ (林贤青), Chen H-Z(陈惠哲). Analysis of status and constraints of rice production in the world. Sci Agric Sin (中国农业 科学), 2010, 43 (3): 474-479 (in Chinese with English abstract)

[3] Jiang L-G(江立庚), Gan X-Q(甘秀芹), Wei S-Q(韦善清), Xu $J-Y($ 徐建云), Cao W-X(曹卫星). Accumulation pattern of dry matter, nitrogen, phosphorus, potassium and silicon in rice genotypes and their relationships. Chin J Appl Ecol (应用生态学报), 2004, 15(2): 226-230 (in Chinese with English abstract)

[4] Yin C-Y(殷春渊), Wei H-Y(魏海燕), Zhang Q(张庆), Dai Q-G(戴其根), Huo Z-Y(霍中洋), Xu K(许轫), Zhang S-F(张胜 飞), Hang J(杭杰), Ma Q(马群). Differences and correlations in grain yield, $\mathrm{N}$ uptake and utilization between medium-maturing indica and japonica rice under different $\mathrm{N}$ fertilizer levels. Acta Agron Sin (作物学报), 2010, 36(4): 655-664 (in Chinese with English abstract)

[5] Sun Y-J(孙永健), Sun Y-Y(孙园园), Li X-Y(李旭毅), Zhang R-P(张荣萍), Guo X(郭翔), Ma J(马均). Effects of waternitrogen interaction on absorption, translocation and distribution of nitrogen, phosphorus, and potassium in rice. Acta Agron Sin (作物学报), 2010, 36(4): 655-664 (in Chinese with English abstract)

[6] Liu L-J(刘立军), Xue Y-G(薛亚光), Sun X-L(孙小淋), Wang Z-Q(王志琴), Yang J-C(杨建昌). Effects of water management methods on grain yield and fertilizer-nitrogen use efficiency in rice. Chin J Rice Sci (中国水稻科学), 2009, 23(3): 282-288 (in Chinese with English abstract)

[7] He Y-Q(何园球), Li C-L(李成亮), Wang X-X(王兴祥), Xiong Y-S(熊又升), Shen Q-R(沈其荣). Effect of soil moisture content and phosphorus application on phosphourus uptake by rice cultivated in aerobic soil. Acta Pedol Sin (土壤学报), 2005, 42(4): 628-633 (in Chinese with English abstract)

[8] Ao H-J(敖和军), Wang S-H(王淑红), Zou Y-B(邹应斌), Peng S-B(彭少兵), Cheng Z-W(程兆伟), Liu W(刘武), Tang Q-Y(唐 启源). Characteristics of nutrient uptake and utilization of super hybrid rice under different fertilizer application rates. Sci Agric Sin (中国农业科学), 2008, 41(10): 3123-3132 (in Chinese with English abstract)

[9] Chen X-H(陈新红), Xu G-W(徐国伟), Wang Z-Q(王志琴), Yang $\mathrm{J}-\mathrm{C}($ 杨建昌). Effects of water and nitrogen on nitrogen utilization and nutrient uptake of rice at late stage. Agric Res Arid Areas ( $干$ 旱地区农业研究), 2004, 22(2): 35-41 (in Chinese with English 
abstract)

[10] Xu M-G(徐明岗), Li D-C(李冬初), Li J-M(李菊梅), Qin D-Z(秦 道珠), Yagi K(八木一行), Hosen Y(宝川靖和). Effects of organic manure application combined with chemical fertilizers on nutrients absorption and yield of rice in Hunan of China. Sci Agric Sin (中国农业科学), 2008, 41(10): 3133-3139 (in Chinese with English abstract)

[11] Du Y(杜永), Liu H(刘辉), Yang C(杨成), Wang Z-Q(王志琴), Yang J-C(杨建昌). Characteristics of nutrient absorption in super-high-yielding mid-season and late-maturity japonica rice. Acta Agron Sin (作物学报), 2007, 33(2): 208-215 (in Chinese with English abstract)

[12] Chen X-H(陈新红), Liu K(刘凯), Xu G-W(徐国伟), Wang Z-Q(王志琴), Yang J-C(杨建昌). Effect of nitrogen and soil moisture on nutrient absorption and quality of rice. $J$ Northwest Sci-Tech Univ Agric \&For (Nat Sci Edn)(西北农林科技大学学 报·自然科学版), 2004, 32(3): 15-21 (in Chinese with English abstract)

[13] Pao S-D(鲍士旦). Soil and Agricultural Chemistry Analysis (土 壤农化分析). Beijing: China Agriculture Press, 2000. pp 264-271 (in Chinese)

[14] Ling Q-H(凌启鸿), Zhang H-C(张洪程), Dai Q-G(戴其根), Ding Y-F(丁艳锋), Ling L(凌励), Su Z-F(苏祖芳), Xu M(徐茂), Que J-H(夙金华), Wang S-H(王绍华). Study on precise and quantitative N application in Rice. Sci Agric Sin (中国农业科学), 2005, 38(12): 2457-2467 (in Chinese with English abstract)

[15] Li G-H(李刚华), Zhang G-F(张国发), Chen G-L(陈功磊), Wang S-H(王绍华), Ling Q-H(凌启鸿), Ding Y-F(丁艳锋). Population characteristics of super japonica rice Ningjing 1 and Ningjing 3 and its responses to nitrogen. Acta Agron Sin (作物学报), 2009, 35(6): 1106-1114 (in Chinese with English abstract)

[16] Chen W F, Xu Z J, Zhang W Z, Zhang L B, Yang S R. Creation of new plant type and breeding rice for super high yield. Acta Agron Sin (作物学报), 2001, 27(5): 665-672

[17] Zhang H-S(张洪松), Tadatoshi I(岩田忠寿). Comparison of matter production and nutrition characteristics for japonica hybrid rice and conventional rice. Southwest China J Agric Sci (西 南农业学报), 1995, 8(4): 11-16 (in Chinese with English abstract)

[18] Ying J F, Peng S B, He Q R, Yang H, Yang C D, Visperas R M, Cassman K G. Comparison of high-yield rice in tropical and subtropical environments: I. Determinations of grain and dry matter yields . Field Crops Res, 1998, 57: 71-84

[19] Yang H-J(杨惠杰), Li Y-Z(李义珍), Yang R-C(杨仁崔), Jiang Z-W(姜照伟), Zheng J-S(郑景生). Dry matter production characteristics of super high-yielding rice. Chin J Rice Sci (中国水稻 科学), 2001, 15(4): 265-270 (in Chinese with English abstract)

[20] Yang H-J(杨惠杰), Yang R-C(杨仁崔), Li Y-Z(李义珍), Jiang Z-W(姜照伟), Zheng J-S(郑景生). Yield potential and yield components of super high-yielding rice cultivars. Fujian J Agric Sci (福建农业学报), 2000, 15(3): 1-8 (in Chinese with English abstract)

[21] Yang H-J(杨惠杰), Yang R-C(杨仁崔), Li Y-Z(李义珍), Zheng J-S(郑景生), Jiang Z-W(姜照伟). Determination factor for super-high yield of rice. Fujian J Agric Sci (福建农业学报), 2002, 17(4): 199-203 (in Chinese with English abstract)

[22] Ling Q-H(凌启鸿), Zhang H-C(张洪程), Cai J-Z(蔡建中), Su Z-F(苏祖芳), Ling L(凌励). Investigation on the population quality of high yield and its optimizing control programme in rice. Sci Agric Sin (中国农业科学), 1993, 26(6): 1-11 (in Chinese with English abstract)

[23] Ling Q-H(凌启鸿), Zhang H-C(张洪程), Ding Y-F(丁艳锋), Zhang Y-B(张益斌). Advances in high yielding techniques in rice-Precise and quantitative cultivation. China Rice (中国稻 米), 2005, (1): 3-7 (in Chinese)

[24] Zhang H-C(张洪程), Wu G-C(吴桂成), Wu W-G(吴文革), Dai Q-G(戴其根), Huo Z-Y(霍中洋), Xu K(许轫), Gao H(高辉), Wei H-Y(魏海燕), Huang X-F(黄幸福), Gong J-L(龚金龙). The SOI model of quantitative cultivation of super-high yielding rice. Sci Agric Sin (中国农业科学), 2010, 43(13): 2645-2660 (in Chinese with English abstract)

[25] Zou Y-B(刍应斌), Zhou S-Y(周上游), Tang Q-Y(唐起源). Status and outlook of high yielding cultivation researches on China super hybrid rice. Rev China Agric Sci Technol (中国农业科技导 报), 2003, 5 (1): 31-35 (in Chinese with English abstract)

[26] Kashiwagi T, Hirotsu N, Ujiie K, Ishimaru K. Lodging resistance locus prl5 improves physical strength of the lower plant part under different conditions of fertilization in rice (Oryza sativa L.). Field Crops Res, 2010, 115: 107-115

[27] Yu W-T(宇万太), Ma Q(马强), Zhou H(周杹), Shen S-M(沈善 敏). Productivity and nutrient budget of rice ecosystem in lower reaches of Liaohe River plain under effects of different fertilization patterns. Chin J Ecol (生态学杂志), 2007, 26(9): 1350-1354 (in Chinese with English abstract) 
[28] Ling Q-H(凌启鸿). Theory and Technology of Rice Precision and Quantitative Cultivation (水稻精确定量栽培理论与技术). Beijing: China Agriculture Press, 2007. pp 76-91 (in Chinese)

[29] Li J-H(李景蕻), Li G-H(李刚华), Zhang Y-G(张应贵), Luo Q-R(罗启荣), Yang C-D(杨从党), Wang S-H(王绍华), Liu Z-H(刘 正辉), Wang Q-S(王强盛), Ding Y-F(丁艳锋). Effects of precise and quantitative cultivation on plant type and yield of rice in high altitude and cold ecological area. Sci Agric Sin (中国农业科学), 2009, 42(9): 3067-3077 (in Chinese with English abstract)

[30] Ling Q-H(凌启鸿). Formation and development of theory and technological system of rice cultivation with Chinese characteristics-report for the 100th Anniversary of Chen Yongkang's Birth. Jiangsu J Agric Sci (江苏农业学报), 2008, 24(2): 101-103 (in Chinese with English abstract)

[31] Peng S-B(彭少兵), Huang J-L(黄见良), Zhong X-H(钟旭华), Yang J-C(杨建昌), Wang G-H(王光火), Zou Y-B(邹应斌),
Zhang F-S(张福锁), Zhu Q-S(朱庆森), Buresh R, Witt C. Research strategy in improving fertilizer-nitrogen use efficiency of irrigated rice in china. Sci Agric Sin (中国农业科学), 2002, 35(9): 1095-1103 (in Chinese with English abstract)

[32] Jing Q, Bouman B, Keulen H V, Hengsdijk H, Cao W X, Dai T B. Disentangling the effect of environmental factors on yield and nitrogen uptake of irrigated rice in Asia. Agric Syst, 2009, 98: 177-188

[33] Yoshida H, Horie T. A model for simulating plant $\mathrm{N}$ accumulation, growth and yield of diverse rice genotypes grown under different soil and climatic conditions. Field Crops Res, 2010, 117: 122-130

[34] Peng S B, Buresh R J, Huang J L, Zhong X H, Zou Y B, Yang J C, Wang G H, Liu Y Y, Hu R F, Tang Q Y. Improving nitrogen fertilization in rice by site-specific $\mathrm{N}$ management: a review. Sustain Agric, 2011, 7: 943-952 